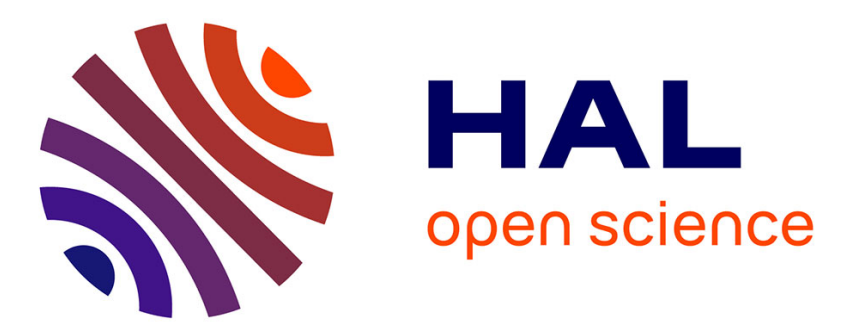

\title{
Mass Resolution Enhancement in Local-Electrode Atom Probes: A Preliminary Study Using Field Emitter
} Arrays

\author{
S. Bajikar, D. Larson, P. Camus, T. Kelly
}

\section{> To cite this version:}

S. Bajikar, D. Larson, P. Camus, T. Kelly. Mass Resolution Enhancement in Local-Electrode Atom Probes: A Preliminary Study Using Field Emitter Arrays. Journal de Physique IV Proceedings, 1996, 06 (C5), pp.C5-303-C5-308. 10.1051/jp4:1996549 . jpa-00254428

\section{HAL Id: jpa-00254428 https://hal.science/jpa-00254428}

Submitted on 1 Jan 1996

HAL is a multi-disciplinary open access archive for the deposit and dissemination of scientific research documents, whether they are published or not. The documents may come from teaching and research institutions in France or abroad, or from public or private research centers.
L'archive ouverte pluridisciplinaire $\mathbf{H A L}$, est destinée au dépôt et à la diffusion de documents scientifiques de niveau recherche, publiés ou non, émanant des établissements d'enseignement et de recherche français ou étrangers, des laboratoires publics ou privés. 


\title{
Mass Resolution Enhancement in Local-Electrode Atom Probes: A Preliminary Study Using Field Emitter Arrays
}

\author{
S.S. Bajikar*, D.J. Larson*,***, P.P. Camus**,*** and T.F. Kelly*,**,*** \\ * Materials Science Program, University of Wisconsin, Madison, WI 53706, U.S.A. \\ ** Department of Materials Science and Engineering, University of Wisconsin, Madison, WI 53706, \\ U.S.A. \\ *** Applied Superconductivity Center, University of Wisconsin, Madison, WI 53706, U.S.A.
}

\begin{abstract}
The use of post-evaporation acceleration as a means to enhance mass resolution in localelectrode atom probes (LEAP) is experimentally investigated in this study. Gated silicon field emitter arrays which have an extraction electrode-tip structure similar to a LEAP are used to evaporate ions at low voltages which are then accelerated to higher voltages. The study confirms the expected improvement in mass resolution. The mass resolution achieved using this gated field emitter array is however quite poor owing to capacitive coupling between the extraction electrode and the tip which leads to a broadened evaporation pulse. An improved model to relate time-of-flight to ion mass-to-charge ratio is found to be necessary in this atom probe configuration. These findings suggest important design considerations for extraction electrodes for a LEAP.
\end{abstract}

\section{INTRODUCTION}

The use of post-evaporation acceleration of ions has been suggested [1-3] as a means of improving mass resolution in a Local-Electrode Atom Probe (LEAP) [1-4]. In a LEAP, a local extraction electrode is used to select a single tip from within an array of tips formed on a planar sample [5]. It also enables the evaporation of ions at low voltages [1-4] due to its proximity to the sample tip. The improvement in mass resolution by post-acceleration of ions is attained by lowering the relative energy dispersion of the evaporated ions. The energy dispersion of the ions at evaporation is a small fraction of the evaporation voltage and depends on the pulse shape. By accelerating the ions to a higher voltage after evaporation, this energy dispersion is reduced to a small fraction of the total ion energy and the mass resolution is thereby improved. The improvement in mass resolution has been modeled and analyzed by Kelly et al. [1] and Bajikar et al. [3] and is dependent on the geometry and distribution of the electric field in the post-evaporation or secondary acceleration region of the instrument [3].

In this paper, we experimentally verify mass resolution improvement achieved by the postacceleration of ions in a LEAP configuration. The experiments are performed using gated silicon field emitter arrays which have an extraction electrode and tip structure similar to a LEAP. This enables mass resolution experiments to be performed without the need for positioning and aligning the extraction electrode with the sample tips. Atom probe studies were previously carried out by Huang et al. on similar 
devices [6] but these studies were focused on characterizing the tips themselves and made no attempt to examine or modify mass resolution.

\section{EXPERIMENTAL}

Gated field emitter arrays were fabricated on n-doped silicon substrates with a resistivity of $0.01 \Omega \mathrm{cm}$. Tips were formed by plasma etching and were sharpened by oxidation. A self-aligned gate technique was used to fabricate the gate (extraction) electrode [7]. The tip-toelectrode distance $(1 \mu \mathrm{m})$ is set by the thickness of the oxide layer separating them. The arrays ( $32 \mathrm{X} 32)$ consisted of 1024 tips each. A scanning electron microscope (SEM) image of the array can be seen in Fig. 1. These emitters showed current-voltage characteristics typical of field emitter arrays with emission (3nA) beginning at a gate-to-tip voltage of about $100 \mathrm{~V}$.

The emitters were tested in a standard voltage-

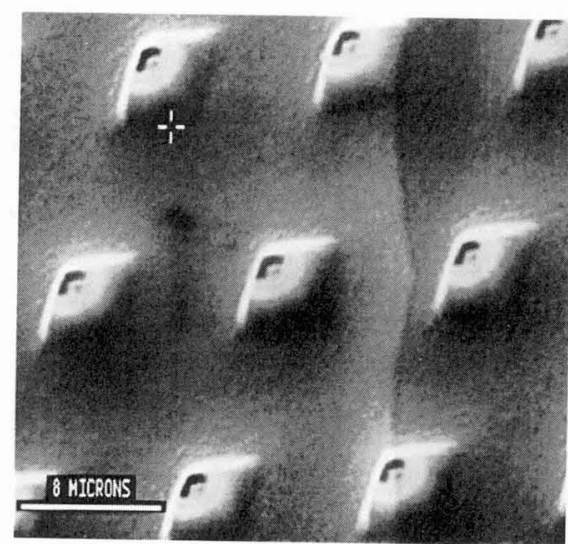

Figure 1. SEM image of silicon field emitter array used for the atom probe studies.

pulsed atom probe setup with minor modifications. An extra voltage lead connecting to the gate (extraction) electrode was provided. The experimental set up for these tests is shown in Fig. 2 . The post-accelerating voltage was applied between the detector $(70 \mathrm{~mm}$ diameter and held at chamber potential) and the extraction electrode. Both the extraction electrode and the emitters (tips) were held at the same (post-accelerating) voltage between evaporation pulses with no stand-off bias and the entire evaporation field/voltage was generated by the pulse. The evaporation pulse was coupled to

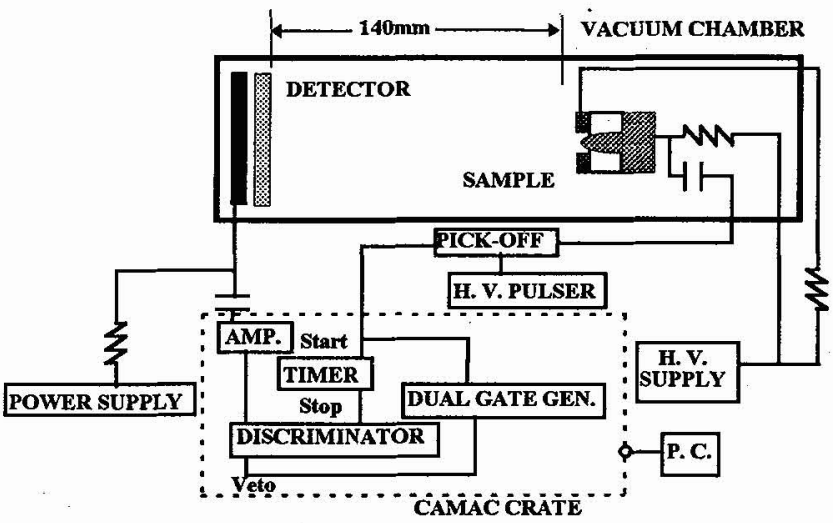

Figure 2. Schematic diagram of experimental set-up for atom probe studies on the field emitter arrays.

the tips through a capacitor. Pressure during testing was about $1 \times 10^{-8}$ Torr and the tips were at room temperature. Two devices were tested (denoted I \& II). Time-of-flight spectra were acquired at a couple of different post-accelerating voltages. The effect of post-acceleration on evaporation rates was also examined.

\section{RESULTS \& DISCUSSION}

\subsection{Pulse Distortion and Gate Pulsing}

It was observed (using an oscilloscope) that the extraction electrode-to-tip pulse is severely distorted and attenuated. While the pulser puts out a $10 \mathrm{~ns}$ pulse with-a one nanosecond rise time, the pulse measured on the electrode was much broader, triangular in profile and about $20 \mathrm{~ns}$ wide at the base. Moreover the pulse was transmitted to the electrode as well. The peak amplitude of the tip-to-electrode pulse was 
about measured to be $40 \mathrm{~V}$ for every $500 \mathrm{~V}$ pulser output voltage, but the accuracy of this measurement is uncertain. This number and the observed pulse shape is however consistent with the large time constant $\left(\sim 10^{-4} \mathrm{~s}\right)$ associated with the large tip-electrode capacitance (hundreds of $\left.\mathrm{pf}\right)$ and the $1 \mathrm{M} \Omega$ resistance added to the circuit to prevent large current pulses from being transmitted to the post-acceleration power supply. The transmission of the pulse to the gate creates a larger post-acceleration field on the ions for the duration of the pulse and has to be considered when calculating mass-to-charge ratios from time spectra. The poor pulse shape may also lead to a time and energy dispersion in the evaporated ions that is greater than that normally encountered in atom probes [7].

\subsection{Post-Accelerating Field Penetration}

Field evaporation studies show that the postacceleration field has a substantial effect on the tip field. This can be expected from the structure of the gate electrode on the arrays. The tip apexes are about even with the gate apertures and are exposed to the postacceleration field. The relative influence of the gate and post-accelerating voltages on the tip field was estimated by measuring the gate voltage needed to produce an evaporation rate of 1 ion per 200 pulses at different post-acceleration voltages. This is shown in Fig. 3 for device I. Extrapolating the data shows that a postaccelerating voltage of about $35,000 \mathrm{~V}$ is equivalent to a gate voltage of $75 \mathrm{~V}$ in terms of the tip field achieved. These evaporation voltages seem low when compared to the field emission voltage, but as mentioned earlier

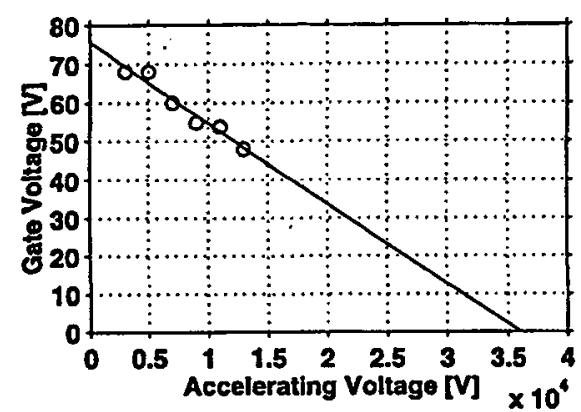

Figure 3. Combinations of gate and postacceleration voltages yielding an evaporation rate of 1 ion/200 pulses for device I.

the exact value of the pulse voltage is somewhat uncertain. However other studies on field emitter arrays [6] have also shown extremely low ratios of evaporation to emission voltage.

This field penetration makes it difficult to control the extraction and evaporation voltages independently. This can affect the mass resolution achieved since it varies the portion of the pulse over which evaporation occurs. Data was therefore acquired at similar evaporation rates under different gate and post-accelerating voltage combinations in order to examine mass resolution.

\subsection{Time-of-flight Spectra}

Field evaporation mass spectra were collected at different post-accelerating voltages. Since it is not possible to independently set both the gate and post-acceleration voltages, the gate voltage was set so as to produce an evaporation rate of 1 detected atom per 25-50 voltage pulses at the different postaccelerating voltages. Acquiring spectra at higher evaporation rates resulted in very wide peaks and no significant improvement in mass resolution. Spectra were acquired from device II first at a postacceleration voltages of $9,000 \mathrm{~V}$ and then at $4,500 \mathrm{~V}$ with gate pulse voltages of $80 \mathrm{~V}$ and $95 \mathrm{~V}$ and are shown in Fig. 4. Even though time dispersion at evaporation is uncertain, the spectrum has been binned in Ins channels. As expected, all peaks have shifted to shorter times at the higher post-acceleration voltage. The relative amplitudes of the peaks is not constant. This may be due to the fact that the spectra were acquired at different times and evaporation may be occurring from different tips owing to 
blunting of active tips with time. The peak labeled "Si" (explained in section 3.4) appears to be a dominant feature in both these spectra. This peak is therefore chosen for the purposes of characterizing mass resolution.

\subsection{Interpretation of Time-of-flight Spectra}

In a conventional voltage-pulsed atom probe, the time-of-flight is normally related to the mass/charge ratio by equating the potential energy lost by the ion to the gain in kinetic energy and determining the speed assuming instantaneous acceleration. Incomplete acceleration of ion to the full pulse voltage is corrected by scaling down the evaporation voltage $\left(V_{e x}\right)$ by the " $\beta$ " factor (i.e. $V_{e x}<\beta$. $V_{e x}$ ) in this calculation [8]. A similar approach in the case of the field emitter array data fails to result in spectra that will match prior results [6]. Using values of $\beta$ between 1 and 0 results in moving the "Si" peak $(28$ amu) from about 40 to $35 \mathrm{amu}$ and a similar shift for (a)

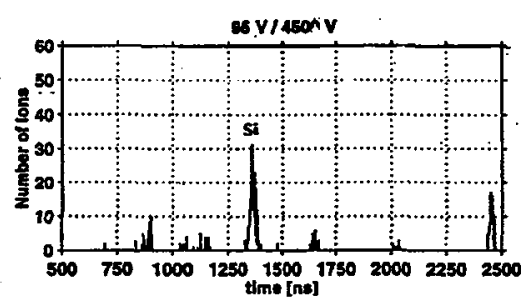

(b)

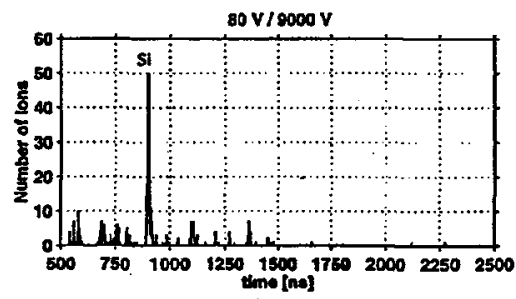

Figure 4. Time-of-flight spectrum acquired at (a) $95 \mathrm{~V} / 4,500 \mathrm{~V}$ and (b) $80 \mathrm{~V} / 9,000 \mathrm{~V}$ for device II all other peaks. The inadequacy of this approach is believed to be due to field in the post-acceleration region being more uniform than in a conventional atom probe resulting in longer flight times. This field will be affected by the details of the vacuum chamber, sample holder and detector set up.

To account for this effect a better model [3] has to be used. This model takes into account realistic field profiles in the post-acceleration region of the instrument. The mass-to-charge ratio is related to the time-of-flight by:

$$
\frac{\mathrm{m}}{\mathrm{n}}=\frac{1.91 \times 10^{-4} \cdot \mathrm{V}_{\mathrm{s}} \cdot \mathrm{t}^{2}}{\mathrm{~L}_{\mathrm{s}}^{2}} \mathrm{~K}(\rho, \lambda, v)
$$

where $\quad K=\left\{\frac{\lambda}{\sqrt{v}}+I\right\}^{-2}$ is a correction factor which accounts for the field distribution in the secondary accelerating region of the instrument and

$$
I=\int_{0}^{1} \frac{d \zeta}{\sqrt{\frac{1}{2} \cdot \frac{1}{\ln [\tan (\alpha)]} \cdot \ln \left(\frac{\sqrt{\rho}+\zeta-1}{\sqrt{\rho}-\zeta+-1} \cdot \frac{\sqrt{\rho}+1}{\sqrt{\rho}-1}\right)+v}} \quad \text { and } \alpha=\cos ^{-1}\left(-\sqrt{\frac{\rho}{1+\rho}}\right)
$$

$\rho$ is a parameter characterizing the field distribution or non-uniformity. $1<\rho<\infty$ with $\rho=1$ corresponding to a constant field and $\rho=\infty$ corresponding to instantaneous acceleration. $v$ is the ratio of the secondary accelerating voltage $\left(\mathrm{V}_{\mathrm{s}}\right)$ to the extraction voltage; and $\lambda$ is the ratio of the length of the extraction electrode $\left(L_{e}\right)$ to the length of the secondary accelerating zone $\left(L_{s}\right)$ in $\mathrm{cm} ; \mathrm{m}$ is the mass of the ion in amu; $n$ is the charge of the ion and $t$ is the flight time in $n s$.

The transmission of the evaporation pulse to the gate/extraction electrode further complicates the application of this model to the above results. Since the electrode length is short ( 0 as seen in Fig. 1), ions will be in the post-accelerating region before the pulse is over. This will result in a greater postacceleration field for the ion during the pulse than the steady state post-acceleration field. The consequent reduction in the time-of-flight may be taken into account by increasing the post-accelerating 
voltage. Alternately, since the distance covered by the ion during the pulse is quite small, this excess acceleration in the post-acceleration region is most easily accounted in the " $\beta$ " term itself. Since $\beta$ now accounts for both incomplete acceleration to $V_{e x}$ as well as excess acceleration in the post-acceleration region during the pulse it does not have to be limited to values less than one. Moreover $\beta$ should now be an increasing function of the post-accelerating voltage. Obtaining unique values for both $\beta$ and the " $\rho$ " parameters from the experimental data is difficult. A satisfactory solution can be obtained by arbitrarily setting $\beta=1$ at post-acceleration voltage $4,500 \mathrm{~V}$ leading to a $\rho$ value of 1.2 . The $\beta$ factor at $9,000 \mathrm{~V}$ then has to be 5 , increasing with post-accelerating voltage as expected. This trend of increasing $\beta$ with postacceleration voltage is also supported by data acquired at variable evaporation rates.

The mass spectra generated from the time-of-flight data in Fig. 4 are shown in Fig. 5. The major peaks in the spectrum can be identified as $\mathrm{C}^{+1}(12), \mathrm{H}_{2} \mathrm{O}^{+1}(18), \mathrm{Si}^{+1}(28)$ and $\mathrm{CO}_{2}^{+1}(44)$ as indicated in Fig. 5. The spectrum matches quite well with the silicon field emitter array spectrum reported earlier by Huang et al. [6], with the dominant evaporating species being $\mathrm{Si}^{+1}$ in addition to several adsorbed gases. In contrast, voltage pulsed atom probe studies on single silicon tips have yielded $\mathrm{Si}^{+2}$ and $\mathrm{SiH}_{\mathbf{x}}{ }^{+2}$ as the major evaporating species with smaller amounts of $\mathrm{Si}^{+1}[9]$. The reason for this difference is uncertain.

(a)

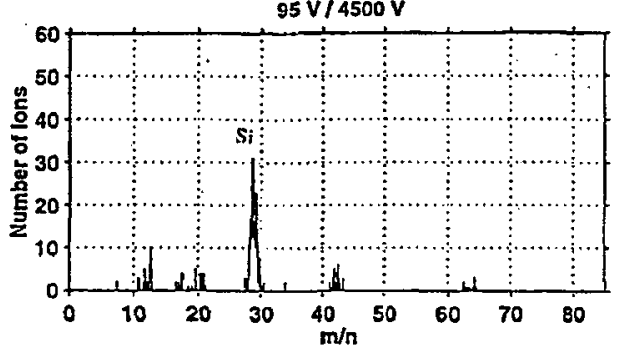

(b)

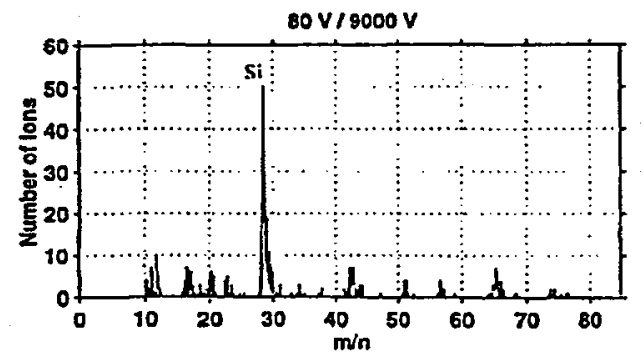

Figure 5. Mass spectrum obtained from the time-of-flight spectrum at (a) $95 \mathrm{~V} / 4,500 \mathrm{~V}$ and (b) $80 \mathrm{~V} / 9,000 \mathrm{~V}$.

\subsection{Mass Resolution}

Mass resolution was determined by examining the $\mathrm{Si}^{+1}$ peak in the spectra. The spectra had a similar number of ions in this peak: 562 at $95 / 4,500 \mathrm{~V}$ and 570 at $80 / 9,000 \mathrm{~V}$. A blow-up of the mass spectrum of this peak normalized to a peak height of 100 is shown in Fig. 6. The shape of the peak is different from that normally encountered with atom probes and can be attributed to the broad pulses used to trigger evaporation. While it may appear that there are two peaks in this region, it should be noted that the two maxima are separated by less than lamu and that this separation is different at different postaccelerating voltages. This is therefore assumed to a single peak and used to characterize mass resolution. The improvement in mass resolution is clear on examining Fig. 6 (a) and (b). The FWHM decreases from $1.2 \mathrm{amu}$ to $0.4 \mathrm{amu}$ when the post-acceleration voltage is increased from $4,500 \mathrm{~V}$ to $9,000 \mathrm{~V}$ and the mass resolution increases from $1 / 23$ to $1 / 70$ by doubling the post-acceleration from $4,500 \mathrm{~V}$ to $9,000 \mathrm{~V}$. The attained mass resolution, which is determined by both the energy dispersion and time spread at evaporation is still quite poor. Post-acceleration only compensates for the former cause. 
(a)

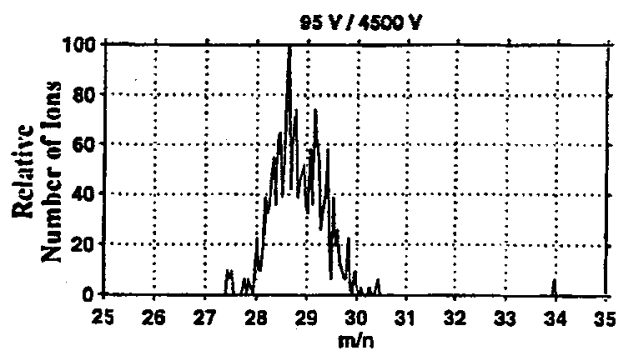

(b)

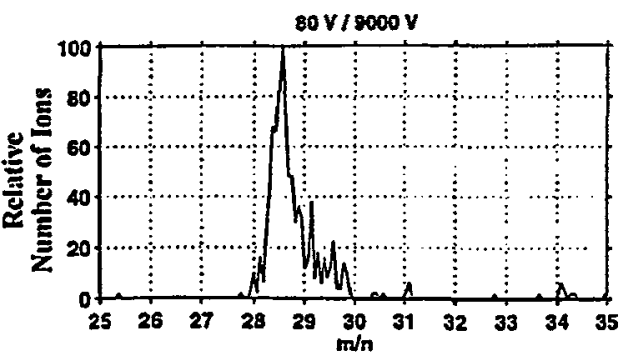

Figure 6. Si peak in mass spectrum normalized to a peak height of 100 at (a) $95 \mathrm{~V} / 4,500 \mathrm{~V}$ and (b) $80 \mathrm{~V} / 9000 \mathrm{~V}$.

\section{CONCLUSIONS}

The field emitter arrays proved to be reliable evaporation sources capable of evaporating thousands of ions at low voltage without failure. The post-acceleration field was found to have a substantial effect on the tip fields which made the independent control of the extraction and post-acceleration voltages impossible. The extraction electrode in a LEAP should therefore be long enough to isolate the tip from the post-acceleration field. Mass-to-charge ratios of the evaporated ions could be determined from the time-of-flight spectra using the model developed by Bajikar et al. [3]. Pulsing of the gate electrode during evaporation pulses was found to result in flight times shorter than expected from the steady state post-accelerating field. This led to an effective $\beta$ value greater than 1 in some cases. The magnitude of $\beta$ was found to be proportional to the post-accelerating voltage. A sufficiently long extraction electrode will eliminate this effect if the ions do not exit it during the duration of the pulse. The size and shape of the extraction electrode also needs to be designed to reduce the capacitance between the it and the tip and avoid undesired transmission of the pulse to the tip. The mass spectra obtained indicated that the tips were contaminated with $\mathrm{C}, \mathrm{H}_{2} \mathrm{O}, \mathrm{CO}_{2}$, etc., and were similar to previously reported atom probe spectra of silicon field emitter arrays. Mass resolution could be improved from $1 / 23$ to $1 / 70$ by increasing the postaccelerating voltage from $4,500 \mathrm{~V}$ to $9,000 \mathrm{~V}$.

\section{Acknowledgments}

This work is sponsored by National Science Foundation under grant \# DMR-8911332 (Drs. L. Haworth, U. Strom and C. Huber).

\section{References}

[1] T. F. Kelly, P. P. Camus, D. J. Larson, L. M. Holzmann and S. S. Bajikar, Ultramicroscopy 62 (1996) 29.

[2] T. F. Kelly, P. P. Camus, D. J. Larson, L. M. Holzmann and S. S. Bajikar, U.S. Patent 5,440,124, (1995).

[3] S. S. Bajikar, D. J. Larson, P. P. Camus and T. F. Kelly, Ultramicroscopy in press (1996).

[4] O. Nishikawa and M. Kimoto, App. Surf. Sci. 76/77 (1994) 424.

[5] D. J. Larson, C-M. Teng, P. P. Camus, and T. F. Kelly, Appl. Surf. Sci. 87/88 (1995) 446.

[6] M. Huang, R. A. D. Mackenzie, T. J. Godfrey and G. D. W. Smith, J. Vac. Sci. Technol. B 12, (1994) 713.

[7] S. S. Bajikar, Ph.D. Thesis, University of Wisconsin-Madison, Madison, WI, USA (1996) 88.

[8] M. K. Miller and G. D. W. Smith, "Atom Probe Microanalysis - Principles and Applications to Materials Problems", Materials Research Society (1989).

[9] C. R. M. Grovener and G. D. W. Smith, Surf. Sci. 123 (1982) L6896. 